\title{
Reduced Model Based Control of Two Link Flexible Space Robot
}

\author{
Amit Kumar ${ }^{1}$, Pushparaj Mani Pathak ${ }^{2}$, Nagarajan Sukavanam ${ }^{1}$ \\ ${ }^{1}$ Department of Mathematics, Indian Institute of Technology Roorkee, Roorkee, India \\ ${ }^{2}$ Department of Mechanical and Industrial Engineering, Indian Institute of \\ Technology Roorkee, Roorkee, India \\ E-mail: tomardma@gmail.com,pushpfme@iitr.ernet.in,nsukvfma@iitr.ernet.in \\ Received January 16, 2011; revised April 11, 2011; accepted April 15, 2011
}

\begin{abstract}
Model based control schemes use the inverse dynamics of the robot arm to produce the main torque component necessary for trajectory tracking. For model-based controller one is required to know the model parameters accurately. This is a very difficult task especially if the manipulator is flexible. So a reduced model based controller has been developed, which requires only the information of space robot base velocity and link parameters. The flexible link is modeled as Euler Bernoulli beam. To simplify the analysis we have considered Jacobian of rigid manipulator. Bond graph modeling is used to model the dynamics of the system and to devise the control strategy. The scheme has been verified using simulation for two links flexible space manipulator.
\end{abstract}

Keywords: Flexible Space Robots, Bond Graph Modeling, Reduced Model Based Controller, Euler-Bernoulli Beam

\section{Introduction}

Space robots are a blend of a vehicle and a robot. It is mechanically more complex than a satellite. The flexible space robot will be useful for space application due to their light weight, less power requirement, ease of maneuverability and ease of transportability. Because of the light weight, they can be operated at high speed. For flexible manipulators flexibility of manipulator have considerable influence on its dynamic behaviors. It is observed by many researchers that fixed-gain linear controllers alone do not provide adequate dynamic performance at high speeds for multi-degrees of-freedom robot manipulators. Out of various schemes studied so far, those involving the calculation of the actuator torque (force) using an inverse dynamics model (the computed torque methods), and those applying adaptive control techniques have been extensively studied, and show the greatest promise.

The traditional control strategy of robot manipulators is completely error driven and shows poor performance at high-speeds, when the high dynamic forces act as disturbances. The current trend is towards model-based control, where the dynamic forces are incorporated in the control strategy as feed forward gains and feed-back compensations along with the servo-controller which is required only to take care of external noise and other factors not included in the dynamic model of the robot. As is expected, the model-based control scheme exhibits better performance, but demands higher computational load in real time. A particular area of model-based control is adaptive control, which is useful when the dynamic parameters of the robot are not well-known a priori. The controller adapts itself during execution of tasks and improves the values of the dynamic parameters.

Murotsu et al. [1,2] proposed control schemes for flexible space manipulator using a virtual rigid manipulator concept. Samanta and Devasia [3] have discussed modeling and control of flexible manipulates using distributed actuator. A technique is presented to analyze the dynamics of flexible manipulators using bond graphs. The nonlinear coupling of large rigid body motion and small elastic vibration of the flexible arms has been taken into consideration in the model. The concept of using distributed piezoelectric transducers for controlling elastic vibration of arms has been incorporated in the analysis. Fardanesh and Rastegar [4] developed an inverse dynamics model based on trajectory pattern method. 
Wang and Gao [5] have reported inverse dynamics model-based control for flexible-link robots, based on modal analysis, i.e., on the assumption that the deformation of the flexible-link can be written as a finite series expansion containing the elementary vibration modes. Leahy et al. [6] worked on robust model based control. They also discussed an experimental case. Fawaz et al. [7] developed a model based real-time virtual simulator of industrial robot in order to detect eventual external collision. The method concerns a model based fault detection and isolation used to determine any lock of motion from an actuated robot joint after contact with static obstacles. Tso et al. [8] worked on a model-based control scheme for robot manipulators employing a variable structure control law in which the actuator dynamics is taken into consideration. Zhu et al. [9] attached an additional model-based parallel-compensator to the conventional model-based computed torque controller which is in the form of a serial compensator to enhance the robustness of robot manipulator control. Qu et al. [10] have discussed robust control of robots by the computed torque law with respect to unknown dynamics by judiciously choosing the feedback gains and the estimates of the nonlinear dynamics. The choices for the constant gains depend only on the coefficients of a polynomial bound of the unknown dynamics. Khosla and Kanade [11] presented the experimental results of the real-time performance of model-based control algorithms. The computed-torque scheme which utilizes the complete dynamics model of the manipulator is compared with the independent joint control scheme which assumes a decoupled and linear model of the manipulator dynamics. Pathak et al. [12] worked on a scheme for robust trajectory control of space robot and this work presents a reduced modal based controller for trajectory control of flexible space robot in work space. Berger et al. [13] carried out the application of bond graph modeling to robots. Special emphasis is placed on adjusting the exact bond graph to allow for valid numerical solutions.

This paper presents a model based controller which requires the information of the base of space robot, link length, joint angle and evaluation of Jacobian. The controller is based on robust overwhelming control of the flexible space manipulator. To illustrate the methodology, an example of a two DOF flexible space robot is considered. The controller is provided with tip velocity information of manipulator. Bond graphs are used to represent both rigid body and flexible dynamics of the link in a unified manner. The advantage of the bond graph is that it is used to model system, in multi energy domain and various control strategies can be devised using this modeling method. SYMBOLS Shakti [14] software is used for bond graph modeling and simulation.

\section{Modeling of Two Arm Flexible Space Robot}

Let us assume that the space robot has single manipulator with revolute joints and is in open kinematic chain configuration. Figure 1 shows the schematic sketch of two arm flexible space robot. In this figure $\{A\}$ represents the absolute frame, $\{V\}$ represents the vehicle frame, $\{0\}$ frame is located in space vehicle at the base of the robot, $\{1\}$ frame is located in first arm at the base of first arm, $\{2\}$ frames is located second joint. The frame $\{t\}$ locates the tip of the robot. Let $L_{1}$ be the length of the first link, $L_{2}$ be the length of second link and $r$ is the distance between the robot base and center of mass $(C M)$ of the space vehicle. Let the first motor is mounted on base and it applies a torque $\tau_{1}$ on first link. Similarly let the second motor is mounted on first link and it applies a torque $\tau_{2}$ on second link. The cross section area of links are assumed to be $A$. The densities of first and second links are $\rho_{1}$ and $\rho_{2}$ respectively. The flexible links are uniform in cross section with flexural rigidities $E I_{1}$ and $E I_{2}$. The flexible link of space robot has been modeled as Euler-Bernoulli beam.

\section{Bond Graph Modeling}

The kinematic analysis of flexible links is performed in order to draw the bond graph as shown in Figure 2. Figure 2 also shows the pad sub model. From the kinematic

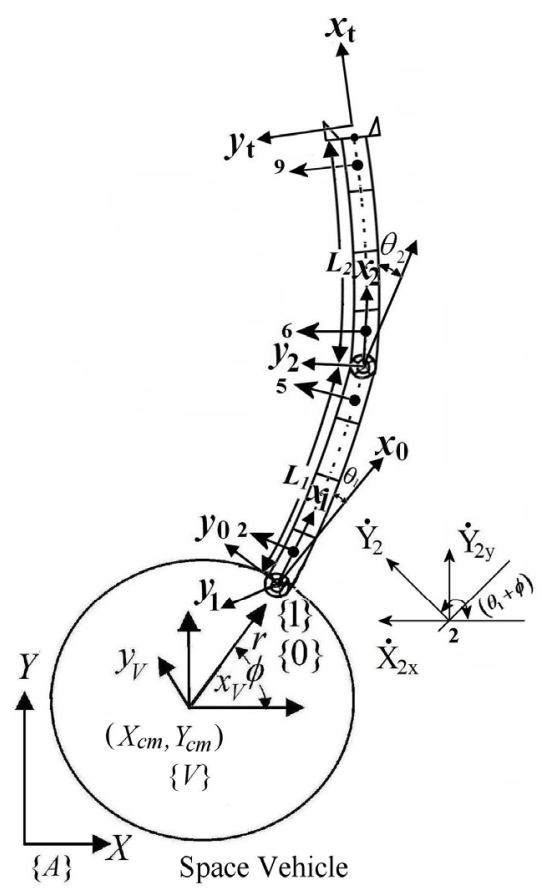

Figure 1. Schematic representation of two arm flexible space robot. 


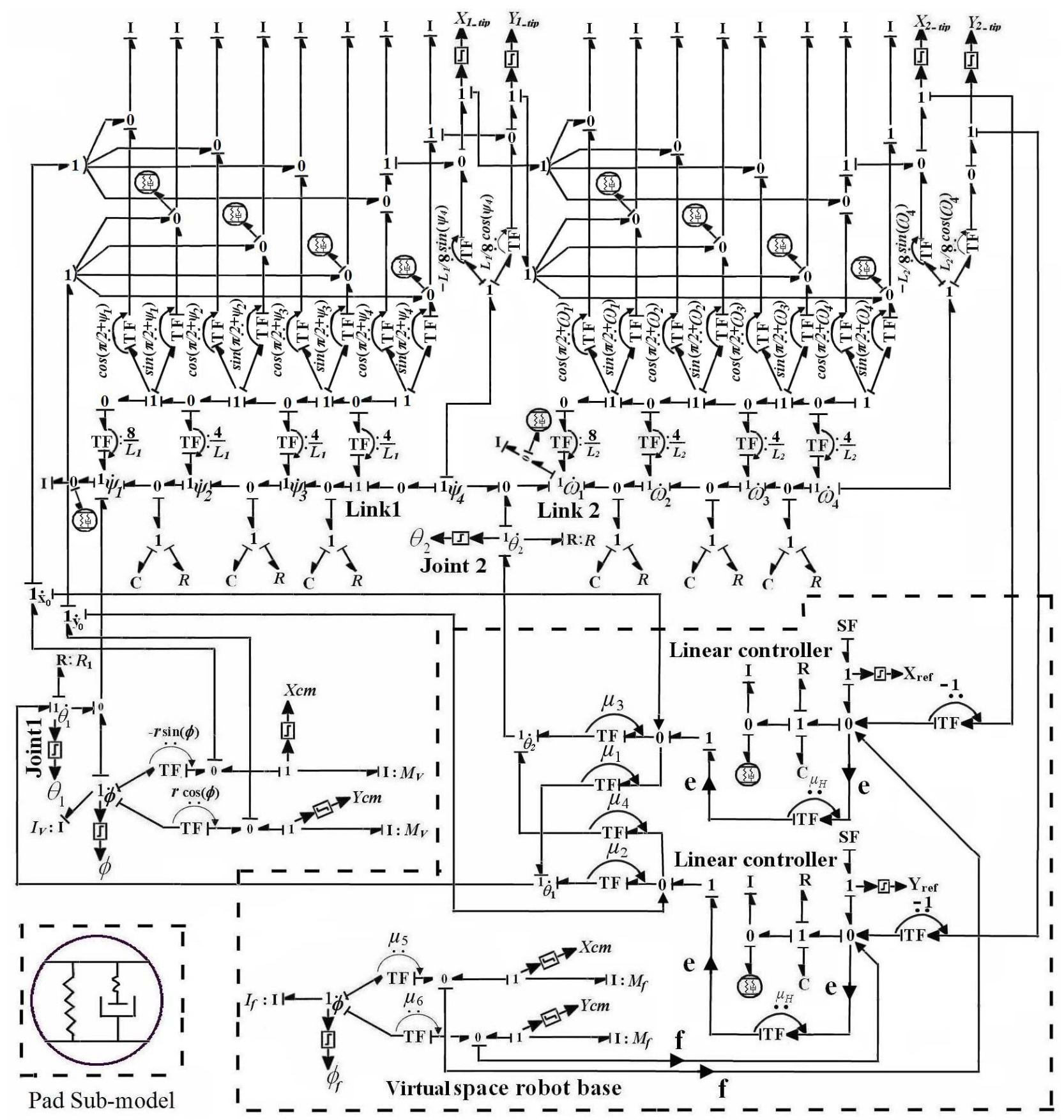

Figure 2. Complete bond graph model of two arm flexible space robot with reduced model based controller.

relations the different transformer moduli used in bond graph model are derived. Two type of motion of the links are considered. First motion is the motion perpendicular to the link and second motion is the rotational motion of the links. The velocities in perpendicular to link is resolved in $X$ and $Y$ direction.

In Figure 2, $M_{V}$ and $I_{V}$ represents the mass of space vehicle and inertia of space vehicle. Let $X_{c m}$ and $Y_{c m}$ be the co-ordinate of the $C M$ of the space vehicle with re- spect to absolute frame. The first and second link is divided into four segments of equal length. The segment angles of first and second link are $\psi_{1}, \psi_{2}, \psi_{3}, \psi_{4}$ and $\omega_{1}$, $\omega_{2}, \omega_{3}, \omega_{4}$ respectively. The segment inertias are attached to " 1 " junction structure corresponding to these velocities. Pads are used for computational simplicity i.e., to avoid the differential causality.

The velocity of the first link at junction 2 in $X$ and $Y$ direction with respect to absolute frame can be evaluated 
as

$$
\begin{aligned}
& \dot{X}_{2 x}=\dot{X}_{c m}-(r \sin \phi) \dot{\phi}+\dot{Y}_{2} \cos \left(\pi / 2+\psi_{1}\right) \\
& \dot{Y}_{2 y}=\dot{Y}_{c m}+(r \cos \phi) \dot{\phi}+\dot{Y}_{2} \sin \left(\pi / 2+\psi_{1}\right)
\end{aligned}
$$

where $\psi_{1}=\theta_{1}+\phi$, and $\dot{Y}_{2}$ is the velocity of the link in direction perpendicular to link at junction 2 . The velocity of the first link tip in $X$ and $Y$ direction with respect to absolute frame can be evaluated as

$$
\begin{aligned}
& \dot{X}_{t_{-} 1}=\dot{X}_{5 x}-\left(L_{1} / 8\right) \sin \left(\psi_{4}\right) \dot{\psi}_{4} \\
& \dot{Y}_{t_{-} 1}=\dot{Y}_{5 y}+\left(L_{1} / 8\right) \cos \left(\psi_{4}\right) \dot{\psi}_{4}
\end{aligned}
$$

where $\dot{X}_{5 x}$ and $\dot{Y}_{5 y}$ are the velocity of the first link at junction 5 in $X$ and $Y$ direction.

The velocity of the second link at junction 6 in $X$ and $Y$ direction with respect to absolute frame can be evaluated as

$$
\begin{gathered}
\dot{X}_{6 x}=\dot{X}_{t_{-} 1}+\dot{Y}_{6} \cos \left(\pi / 2+\omega_{1}\right) \\
\dot{Y}_{6 y}=\dot{Y}_{t_{-} 1}+\dot{Y}_{6} \sin \left(\pi / 2+\omega_{1}\right)
\end{gathered}
$$

where $\dot{Y}_{6}$ is the velocity of second link at junction 6 in the direction perpendicular to link. The velocity of the robot at tip in $X$ and $Y$ direction with respect to absolute frame can be evaluated as

$$
\begin{aligned}
& \dot{X}_{t_{-} 2}=\dot{X}_{9 x}-\left(L_{2} / 8\right) \sin \left(\omega_{4}\right) \dot{\omega}_{4} \\
& \dot{Y}_{t_{-} 2}=\dot{Y}_{9 y}+\left(L_{2} / 8\right) \cos \left(\omega_{4}\right) \dot{\omega}_{4}
\end{aligned}
$$

where $\dot{X}_{9 x}$ and $\dot{Y}_{9 y}$ are velocity of the link in $X$ and $Y$ direction at junction 9 .

From the above kinematic analysis different transformer modulli used in drawing bond graph can be evaluated and are shown in Table $\mathbf{1 .}$

\section{Reduced Model-Based Controller Design}

In work space control the robot is required to track a given end-effector trajectory, this is achieved by using a reduced model based controller. The bond graph model of a two DOF flexible planar space robot controller is shown in Figure 2. The model is represented in the inertial frame. Controller consists of three parts.

1) Space robot virtual base model

2) Overwhelming controller [12]

3) Jacobian.

\begin{tabular}{|c|c|c|}
\hline Description & $X$ Direction & $Y$ Direction \\
\hline Robot Base & $\mathrm{TF}=-r \sin (\phi)$ & $\mathrm{TF}=r \cos (\phi)$ \\
\hline $\begin{array}{l}\text { First Segment of } \\
\text { First Link }\end{array}$ & $\mathrm{TF}=\cos \left(\pi / 2+\psi_{1}\right)$ & $\mathrm{TF}=\sin \left(\pi / 2+\psi_{1}\right)$ \\
\hline $\begin{array}{l}\text { Second Segment } \\
\text { of First Link }\end{array}$ & $\mathrm{TF}=\cos \left(\pi / 2+\psi_{2}\right)$ & $\mathrm{TF}=\sin \left(\pi / 2+\psi_{2}\right)$ \\
\hline $\begin{array}{l}\text { Third Segment } \\
\text { of First Link }\end{array}$ & $\mathrm{TF}=\cos \left(\pi / 2+\psi_{3}\right)$ & $\mathrm{TF}=\sin \left(\pi / 2+\psi_{3}\right)$ \\
\hline $\begin{array}{l}\text { Fourth Segment } \\
\text { of First Link }\end{array}$ & $\mathrm{TF}=\cos \left(\pi / 2+\psi_{4}\right)$ & $\mathrm{TF}=\sin \left(\pi / 2+\psi_{4}\right)$ \\
\hline First Link Tip & $\mathrm{TF}=-\left(L_{1} / 8\right) \sin \left(\psi_{4}\right)$ & $\mathrm{TF}=\left(L_{1} / 8\right) \cos \left(\psi_{4}\right)$ \\
\hline $\begin{array}{l}\text { First Segment of } \\
\text { Second Link }\end{array}$ & $\mathrm{TF}=\cos \left(\pi / 2+\omega_{1}\right)$ & $\mathrm{TF}=\sin \left(\pi / 2+\omega_{1}\right)$ \\
\hline $\begin{array}{l}\text { Second Segment } \\
\text { of Second Link }\end{array}$ & $\mathrm{TF}=\cos \left(\pi / 2+\omega_{2}\right)$ & $\mathrm{TF}=\sin \left(\pi / 2+\omega_{2}\right)$ \\
\hline $\begin{array}{l}\text { Third Segment } \\
\text { of Second Link }\end{array}$ & $\mathrm{TF}=\cos \left(\pi / 2+\omega_{3}\right)$ & $\mathrm{TF}=\sin \left(\pi / 2+\omega_{3}\right)$ \\
\hline $\begin{array}{l}\text { Fourth Segment } \\
\text { of Second Link }\end{array}$ & $\mathrm{TF}=\cos \left(\pi / 2+\omega_{4}\right)$ & $\mathrm{TF}=\sin \left(\pi / 2+\omega_{4}\right)$ \\
\hline Second Link Tip & $\mathrm{TF}=-\left(L_{2} / 8\right) \sin \left(\omega_{4}\right)$ & $\mathrm{TF}=\left(L_{2} / 8\right) \cos \left(\omega_{4}\right)$ \\
\hline
\end{tabular}

\subsection{Space Robot Virtual Base Model}

A virtual base of the space vehicle is considered. To design the model based controller, drawing analogy from the space vehicle, the tip velocity of manipulator in $X$ and $Y$ direction is considered in controller design. It is
Table 1. Transformer moduli for finding velocities at various points in bond graph model.

determined by translational inertia $M_{f}$ of space vehicle. The displacement relation of the robot are given as

$$
\begin{aligned}
X_{\text {tipf }}= & X_{f}+r \cos \phi_{f}+L_{1} \cos \left(\phi_{f}+\theta_{1}\right) \\
& +L_{2} \cos \left(\phi_{f}+\theta_{1}+\theta_{2}\right) \\
Y_{\text {tipf }}= & Y_{f}+r \sin \phi_{f}+L_{1} \sin \left(\phi_{f}+\theta_{1}\right) \\
& +L_{2} \sin \left(\phi_{f}+\theta_{1}+\theta_{2}\right)
\end{aligned}
$$

Here $\phi_{f}$ is the rotation of the space vehicle.

The tip velocity of the robot $\dot{X}_{\text {tipf }}$, and $\dot{Y}_{\text {tipf }}$ due to motion of the space vehicle can be found as,

$$
\begin{aligned}
\dot{X}_{\text {tipf }}= & \dot{X}_{f}-\left[r \sin \phi_{f}+L_{1} \sin \left(\phi_{f}+\theta_{1}\right)\right. \\
& \left.+L_{2} \sin \left(\phi_{f}+\theta_{1}+\theta_{2}\right)\right] \dot{\phi}_{f} \\
& -\left[L_{1} \sin \left(\phi_{f}+\theta_{1}\right)+L_{2} \sin \left(\phi_{f}+\theta_{1}+\theta_{2}\right)\right] \dot{\theta}_{1} \\
& -\left[L_{2} \sin \left(\phi_{f}+\theta_{1}+\theta_{2}\right)\right] \dot{\theta}_{2} \\
\dot{Y}_{\text {tipf }}= & \dot{Y}_{f}+\left[r \cos \phi_{f}+L_{1} \cos \left(\phi_{f}+\theta_{1}\right)\right. \\
& \left.+L_{2} \cos \left(\phi_{f}+\theta_{1}+\theta_{2}\right)\right] \dot{\phi}_{f} \\
& +\left[L_{1} \cos \left(\phi_{f}+\theta_{1}\right)+L_{2} \cos \left(\phi_{f}+\theta_{1}+\theta_{2}\right)\right] \dot{\theta}_{1} \\
& +\left[L_{2} \cos \left(\phi_{f}+\theta_{1}+\theta_{2}\right)\right] \dot{\theta}_{2}
\end{aligned}
$$

If it is assumed that the controller overwhelms the ro- 
bot dynamics, then neglecting the coefficients of $\dot{\theta}_{1}$ and $\dot{\theta}_{2}$ in Equations (11) and (12) we get,

$$
\begin{aligned}
\dot{X}_{\text {tipf }}= & \dot{X}_{f}-\left[r \sin \phi_{f}+L_{1} \sin \left(\phi_{f}+\theta_{1}\right)\right. \\
& \left.+L_{2} \sin \left(\phi_{f}+\theta_{1}+\theta_{2}\right)\right] \dot{\phi}_{f} \\
\dot{Y}_{\text {tipf }}= & \dot{Y}_{f}+\left[r \cos \phi_{f}+L_{1} \cos \left(\phi_{f}+\theta_{1}\right)\right. \\
& \left.+L_{2} \cos \left(\phi_{f}+\theta_{1}+\theta_{2}\right)\right] \dot{\phi}_{f}
\end{aligned}
$$

Using Equation (13) and (14), the transformer modulli $\mu_{5}$, and $\mu_{6}$ shown in bond graph of Figure 2, can be written as,

$$
\begin{gathered}
\mu_{5}=-\left[r \sin \phi_{f}+L_{1} \sin \left(\phi_{f}+\theta_{1}\right)+L_{2} \sin \left(\phi_{f}+\theta_{1}+\theta_{2}\right)\right] \\
\mu_{6}=\left[r \cos \phi_{f}+L_{1} \cos \left(\phi_{f}+\theta_{1}\right)+L_{2} \cos \left(\phi_{f}+\theta_{1}+\theta_{2}\right)\right]
\end{gathered}
$$

One can observe from these expressions that these modulli involve only link lengths and joint angles of the manipulator.

\subsection{Overwhelming Controller}

The linear overwhelming controller [12] is applied to the tip of the space robot model. The overwhelming controller is provided with reference velocity and tip velocity information. The effort signal produced by the controller is magnified by high gain and then the joint torques are evaluated with the help of Jacobian. This joint torque information is fed to different joints.

\subsection{Evaluation of Jacobian}

If we assume that the arms of manipulator are rigid then, kinematic relations for the tip displacement $X_{t i p}, Y_{\text {tip }}$ in $X$ and $Y$ directions can be written as,

$$
\left[\begin{array}{c}
X_{t i p} \\
Y_{\text {tip }}
\end{array}\right]=\left[\begin{array}{c}
X_{C M} \\
Y_{C M}
\end{array}\right]+\left[\begin{array}{c}
r \cos \phi+L_{1} \cos \left(\phi+\theta_{1}\right)+L_{2} \cos \left(\phi+\theta_{1}+\theta_{2}\right) \\
r \sin \phi+L_{1} \sin \left(\phi+\theta_{1}\right)+L_{2} \sin \left(\phi+\theta_{1}+\theta_{2}\right)
\end{array}\right]
$$

The tip angular displacement with respect to absolute frame $X$ axis is given as,

$$
\begin{gathered}
\theta_{\text {tip }}=\phi+\theta_{1}+\theta_{2} \\
{\left[\begin{array}{c}
\dot{X}_{t i p} \\
\dot{Y}_{\text {tip }}
\end{array}\right]=\left[\begin{array}{c}
\dot{X}_{C M} \\
\dot{Y}_{C M}
\end{array}\right]+\left[\begin{array}{cc}
-r \dot{\phi} \sin \phi-L_{1}\left(\dot{\phi}+\dot{\theta}_{1}\right) \sin \left(\phi+\theta_{1}\right)-L_{2}\left(\dot{\phi}+\dot{\theta}_{1}+\dot{\theta}_{2}\right) \sin \left(\phi+\theta_{1}+\theta_{2}\right) \\
r \dot{\phi} \cos \phi+L_{1}\left(\dot{\phi}+\dot{\theta}_{1}\right) \cos \left(\phi+\theta_{1}\right)+L_{2}\left(\dot{\phi}+\dot{\theta}_{1}+\dot{\theta}_{2}\right) \cos \left(\phi+\theta_{1}+\theta_{2}\right)
\end{array}\right]} \\
{\left[\begin{array}{c}
\dot{X}_{t i p} \\
\dot{Y}_{\text {tip }}
\end{array}\right]=\left[\begin{array}{c}
\dot{X}_{C M}-r \dot{\phi} \sin \phi \\
\dot{Y}_{C M}+r \dot{\phi} \cos \phi
\end{array}\right]+\left[\begin{array}{cc}
-L_{1} \sin \left(\phi+\theta_{1}\right)-L_{2} \sin \left(\phi+\theta_{1}+\theta_{2}\right) & -L_{2} \sin \left(\phi+\theta_{1}+\theta_{2}\right) \\
L_{1} \cos \left(\phi+\theta_{1}\right)+L_{2} \cos \left(\phi+\theta_{1}+\theta_{2}\right) & L_{2} \cos \left(\phi+\theta_{1}+\theta_{2}\right)
\end{array}\right]\left[\begin{array}{c}
\dot{\theta}_{1} \\
\dot{\theta}_{2}
\end{array}\right]} \\
+\left[\begin{array}{c}
-L_{1} \sin \left(\phi+\theta_{1}\right)-L_{2} \sin \left(\phi+\theta_{1}+\theta_{2}\right) \\
L_{1} \cos \left(\phi+\theta_{1}\right)+L_{2} \cos \left(\phi+\theta_{1}+\theta_{2}\right)
\end{array}\right]\left[\begin{array}{l}
\dot{\phi}]
\end{array}\right.
\end{gathered}
$$

The Jacobian of the forward kinematics can be calculated in bond graph. For the planar case discussed here this relation can be worked out directly from Equation
For the evaluation of joint torque if we assume that space vehicle rotational velocity $\dot{\phi}$, and $C M$ velocity
$\dot{X}_{C M}, \dot{Y}_{C M}$ are small, then neglecting the first and last term of Equation (18) we get

$$
\begin{aligned}
& {\left[\begin{array}{c}
\dot{X}_{t i p} \\
\dot{Y}_{t i p}
\end{array}\right]=\left[\begin{array}{cc}
-L_{1} \sin \left(\phi+\theta_{1}\right)-L_{2} \sin \left(\phi+\theta_{1}+\theta_{2}\right) & -L_{2} \sin \left(\phi+\theta_{1}+\theta_{2}\right) \\
L_{1} \cos \left(\phi+\theta_{1}\right)+L_{2} \cos \left(\phi+\theta_{1}+\theta_{2}\right) & L_{2} \cos \left(\phi+\theta_{1}+\theta_{2}\right)
\end{array}\right]\left[\begin{array}{c}
\dot{\theta}_{1} \\
\dot{\theta}_{2}
\end{array}\right]} \\
& {\left[\begin{array}{c}
\dot{X}_{t i p} \\
\dot{Y}_{t i p}
\end{array}\right]=[J]\left[\begin{array}{c}
\dot{\theta}_{1} \\
\dot{\theta}_{2}
\end{array}\right]} \\
& \mu_{3}=-L_{2} \sin \left(\phi+\theta_{1}+\theta_{2}\right), \\
& \mu_{4}=L_{2} \cos \left(\phi+\theta_{1}+\theta_{2}\right) \text {. }
\end{aligned}
$$

With the help of Equation (20) various transformer modulli shown in bond graph can be evaluated as

$$
\begin{aligned}
& \mu_{1}=-L_{1} \sin \left(\phi+\theta_{1}\right)-L_{2} \sin \left(\phi+\theta_{1}+\theta_{2}\right) \\
& \mu_{2}=L_{1} \cos \left(\phi+\theta_{1}\right)+L_{2} \cos \left(\phi+\theta_{1}+\theta_{2}\right)
\end{aligned}
$$

\section{Simulation and Results}

It is assumed that initially base rotation; first and second joint angles are 0 radian, 0.5 radian and 0 radian respec- 
tively. Figure 3 shows the initial configuration of the space robot. At the beginning of the simulation overwhelmer initial position is initialized to robot tip position.

Let us assume that the reference displacement command for the robot tip is a circle of radius $R$. Then the tip coordinates will be given as,

$$
\begin{gathered}
X_{\text {ref }}(t)=-R \cos (\omega t)+X_{0} \\
Y_{\text {ref }}(t)=R \sin (\omega t)+Y_{0}
\end{gathered}
$$

Here $\left(X_{0}, Y_{0}\right)$ is the center of the circular reference circle. The corresponding reference velocity command is given by,

$$
\begin{aligned}
& f_{\text {refx }}=R \omega \sin (\omega t) \\
& f_{\text {refy }}=R \omega \cos (\omega t)
\end{aligned}
$$

At the start of simulation the reference trajectory is initialized to tip trajectory. The parameters used for

\begin{tabular}{|c|c|}
\hline Parameter & Value \\
\hline Modulus of Elasticity & $E=70 \times 10^{9} \mathrm{~N} / \mathrm{m}^{2}$ \\
\hline Link Length & $L_{1}=0.5 \mathrm{~m} ; L_{2}=0.6 \mathrm{~m}$ \\
\hline Link Inertia & $\begin{aligned} I_{1} & =6.609 \times 10^{-13} \mathrm{~m}^{4}, \\
I_{2} & =6.609 \times 10^{-13} \mathrm{~m}^{4}\end{aligned}$ \\
\hline $\begin{array}{l}\text { Location of Robot Base } \\
\text { from Vehicle } C M\end{array}$ & $r=0.1 \mathrm{~m}$ \\
\hline Mass of the First Link & $m_{1}=1.0 \mathrm{~kg}$ \\
\hline $\begin{array}{l}\text { Cross Section Area of First } \\
\text { and Second Link }\end{array}$ & $\begin{array}{l}A_{1}=6.288 \times 10^{-5} \mathrm{~m}^{2} \\
A_{2}=6.288 \times 10^{-5} \mathrm{~m}^{2}\end{array}$ \\
\hline Joint Resistances & $\begin{array}{l}R_{1}=0.001 \mathrm{Nm} /(\mathrm{rad} / \mathrm{s}), \\
R_{2}=0.0001 \mathrm{Nm} /(\mathrm{rad} / \mathrm{s})\end{array}$ \\
\hline Mass of Base & $M=5.0 \mathrm{~kg}$ \\
\hline $\begin{array}{l}\text { Moment of Inertia of Space } \\
\text { Vehicle }\end{array}$ & $I_{V}=1.0 \mathrm{~kg}-\mathrm{m}^{2}$ \\
\hline Gain Value & $\mu_{\mathrm{H}}=8.0$ \\
\hline Motor Inertias & $\begin{aligned} I_{m 1} & =0.001 \mathrm{~kg} \cdot \mathrm{m}^{2}, \\
I_{m 2} & =0.001 \mathrm{~kg} \cdot \mathrm{m}^{2}\end{aligned}$ \\
\hline Controller & $M_{\mathrm{C}}=0.001, K_{\mathrm{C}}=0.241, R_{\mathrm{C}}=0.13$ \\
\hline $\begin{array}{l}\text { Internal Damping Coeffi- } \\
\text { cient for Material }\end{array}$ & $R_{\text {int }}=0.01 \mathrm{~N} /(\mathrm{m} / \mathrm{s})$ \\
\hline Reference Circle Radius & $R=0.400 \mathrm{~m}$ \\
\hline $\begin{array}{l}\text { Input Reference Angular } \\
\text { Velocity }\end{array}$ & $\omega=1.0 \mathrm{rad} / \mathrm{s}$ \\
\hline Density of Aluminum & $\rho=2700 \mathrm{~kg} / \mathrm{m}^{3}$ \\
\hline
\end{tabular}
simulation are shown in Table 2. The simulation is carried out for 1.56 seconds.

Table 2. Parameters and values used for simulation.

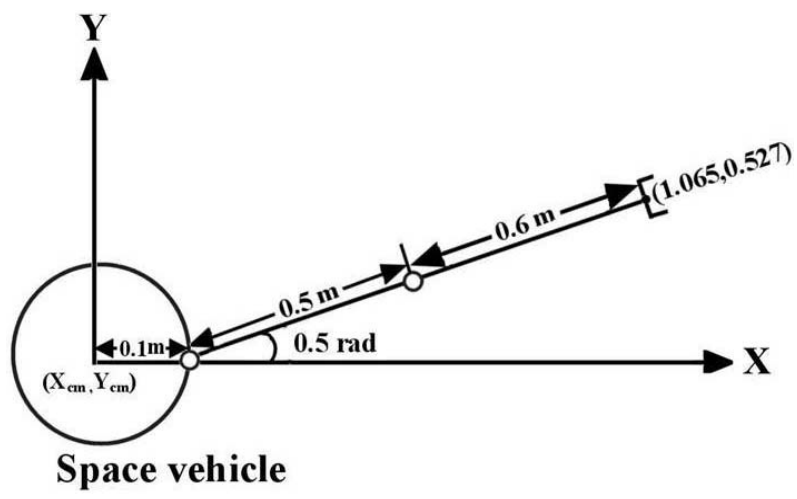

Figure 3. Initial configuration of two arm flexible space robot.

Figure 4(a) shows the plot for reference trajectory in $x$ direction, Figure 4(b) shows the plot reference trajectory in $y$ direction, Figure 4(c) shows the plot for tip trajectory in $x$ direction, and Figure 4(d) shows the plot for tip trajectory in $y$ direction. From Figures 4(a) and (c) it is seen that tip follows the reference trajectory in $x$ direction and from Figures 4(b) and (d) it is also seen that tip follows trajectory in $y$ direction effectively. Due to the nature of the flexible link tip vibration are observed. Figure 5(a) shows the plot of base rotation with respect to time. The base of the space vehicle rotates between the points 0.0 to -0.03 as the space robot is free floating. Figure 5(b) shows the plot of first joint angle with respect to time. Figure 5(c) shows the plot of second joint angle with respect to time Figure 5(d) shows the plot of $C M$ of space vehicle in $x$ direction with respect to time. Figure 5(e) shows the plot $C M$ of space vehicle in y direction with respect to time. Figure 5(f) shows the plot of $C M$ trajectory of space vehicle. Figures 5(d), (e) and (f) it is seen that there is continues movement in base of space robot as it is free floating.

Figure 6 shows the tip trajectory error plot. The error is taken as the difference between reference and actual tip position. Figure 6(a) shows the error plot for $X$ tip position with respect to time. Figure 6(b) shows the error plot for $Y$ tip position with respect to time. From the simulation we see that initially error increases with time but after some time it start reducing.

\section{Conclusions}

In this paper we presented a novel reduced model based controller for trajectory control of flexible space robot in work space. Euler Bernoulli beam model is used to model flexible link. Reduced model based controller consists of three parts 1) the model of the virtual base of space vehicle 2) an overwhelming controller 3) a Jacobian evaluation to evaluate joint torques. The advantage 


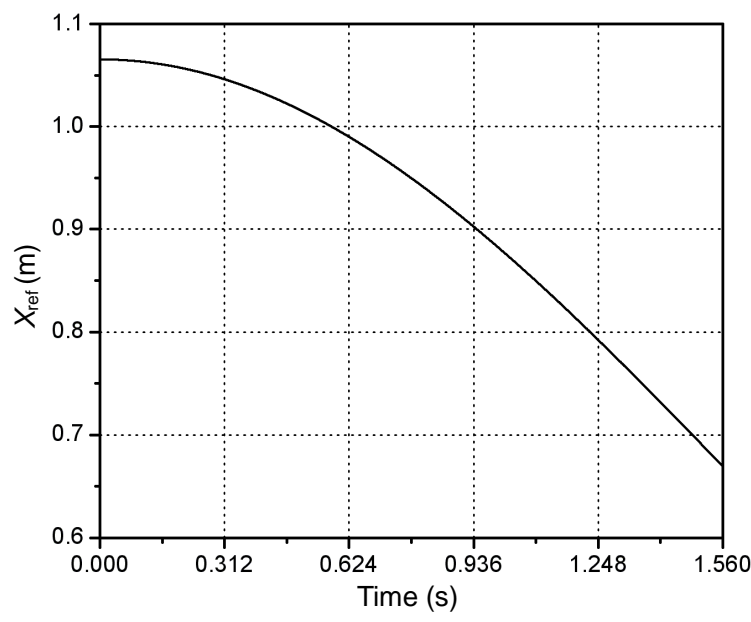

(a)

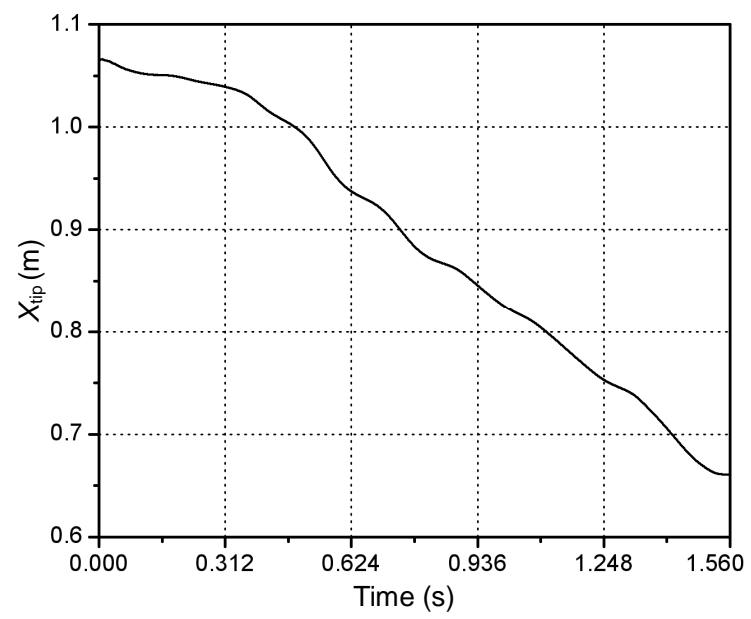

(c)

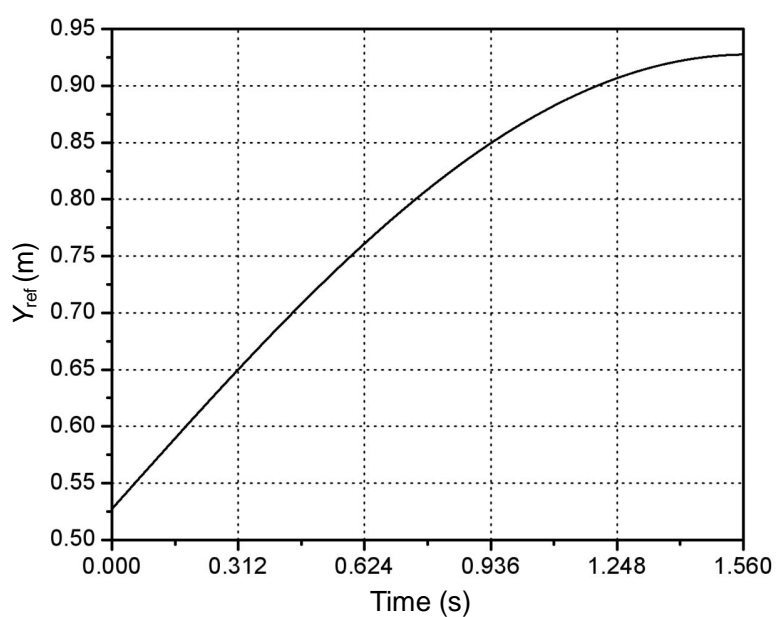

(b)

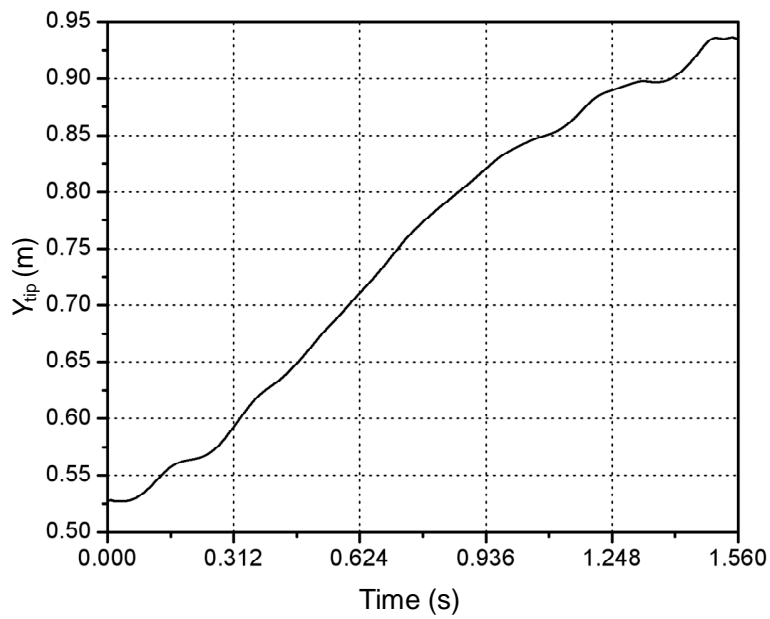

(d)

Figure 4. (a) Reference trajectory in $x$ direction; (b) Reference trajectory in $y$ direction; (c) Tip trajectory in $x$ direction. (d) Tip trajectory in $y$ direction.

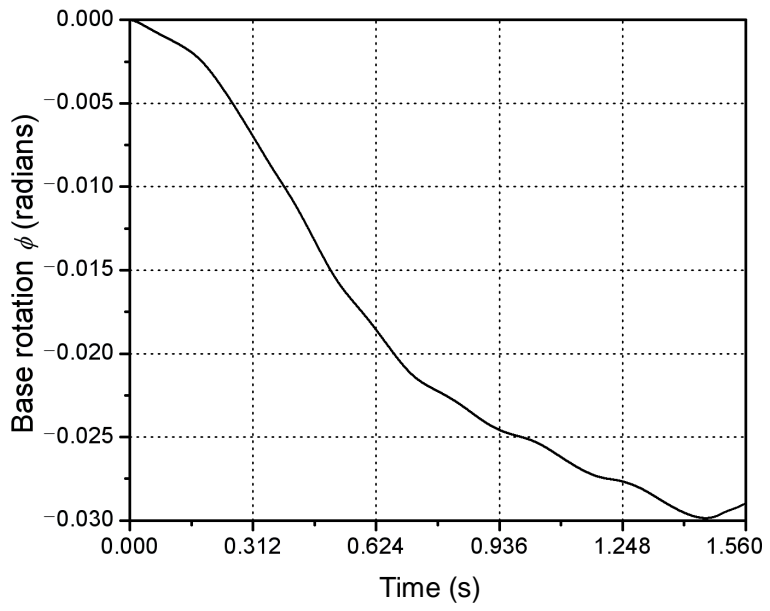

(a)

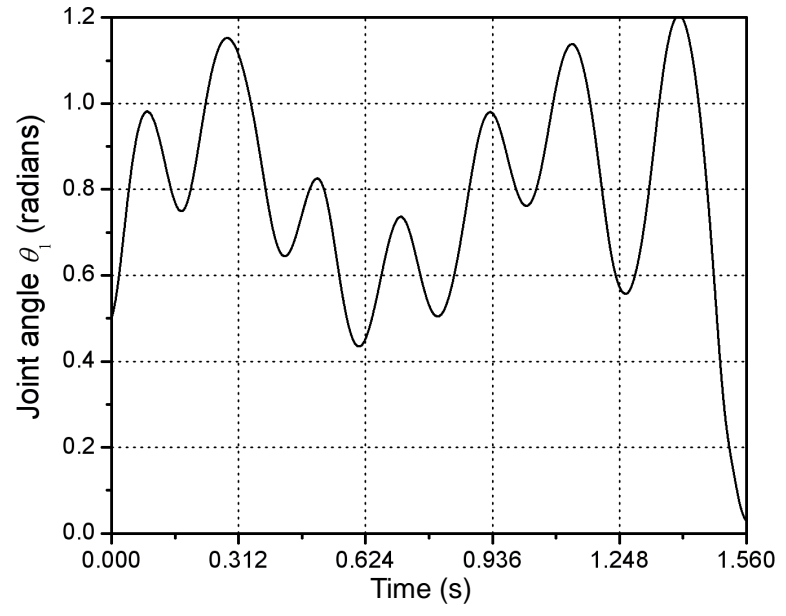

(b) 


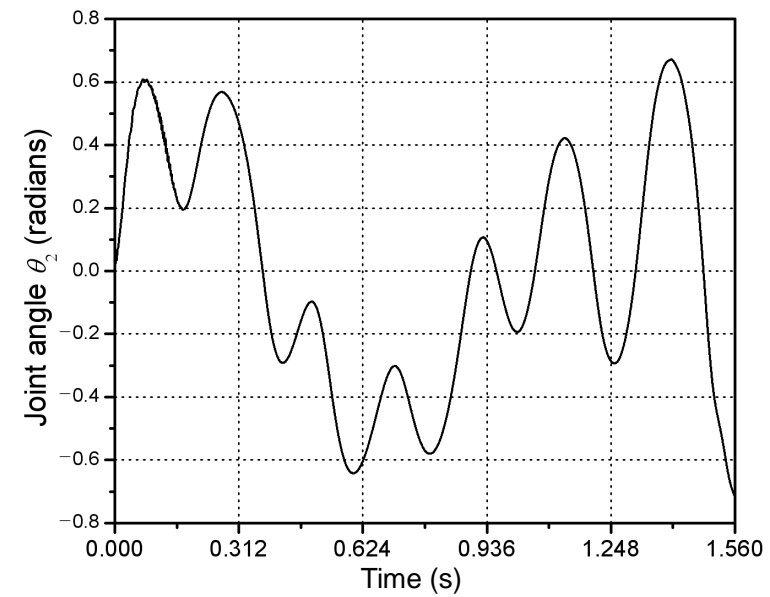

(c)

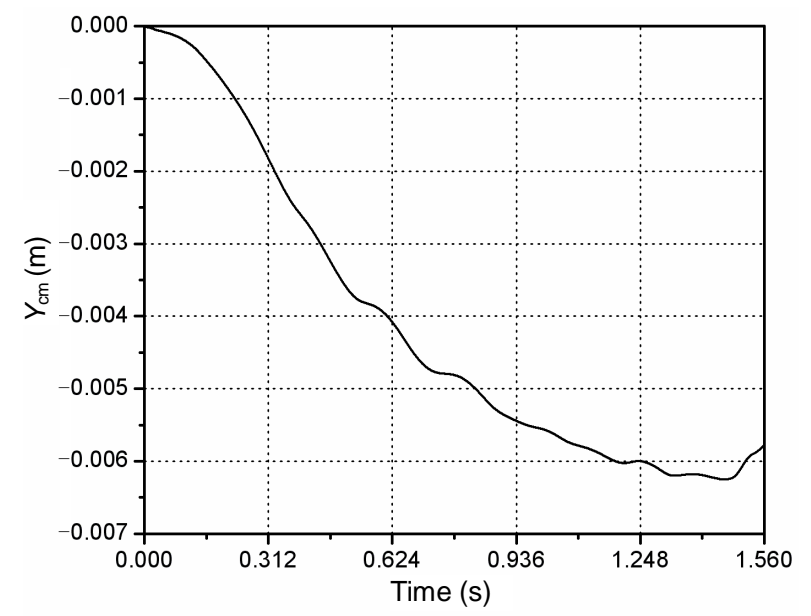

(e)

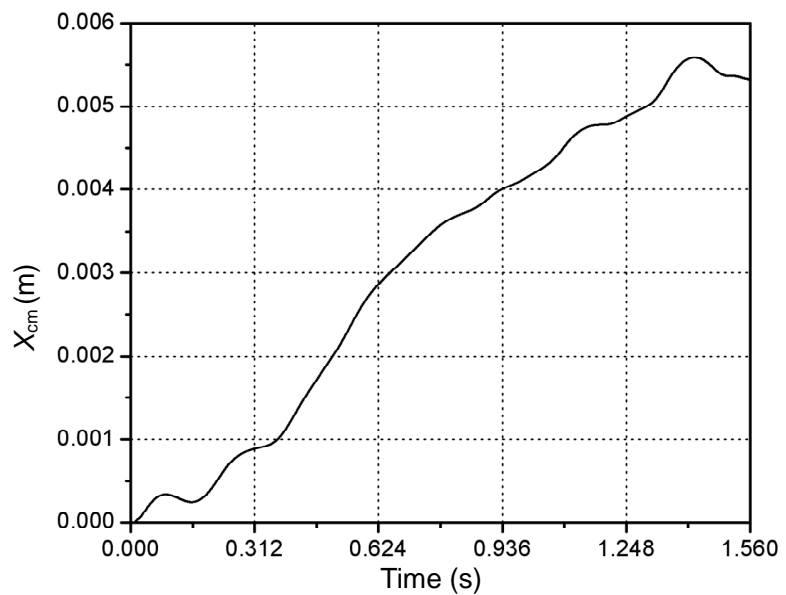

(d)

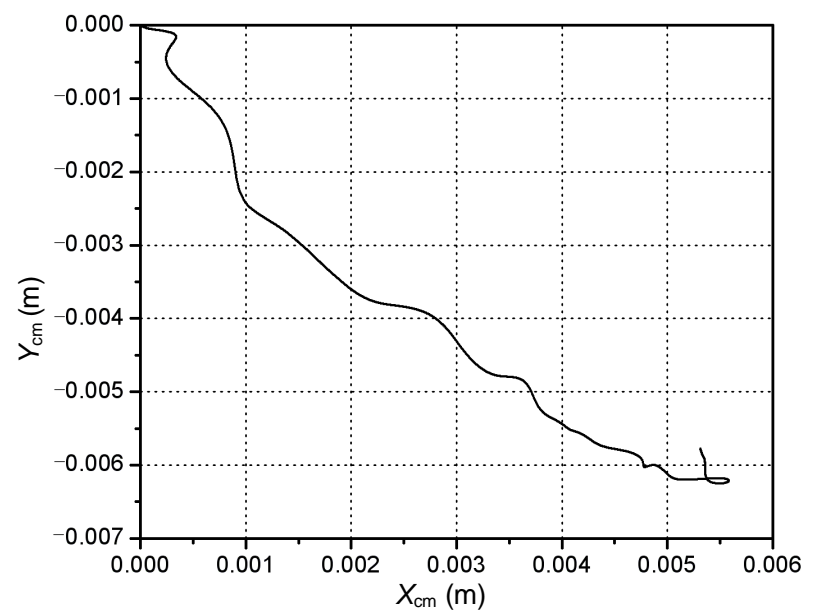

(f)

Figure 5: (a) Plot of base rotation versus time; (b) Plot of first joint angle versus time; (c) Plot of second joint angle versus time; (d) Centre of mass trajectory in $x$ direction; (e) Centre of mass trajectory in $y$ direction; (f) Centre of mass trajectory of space vehicle.

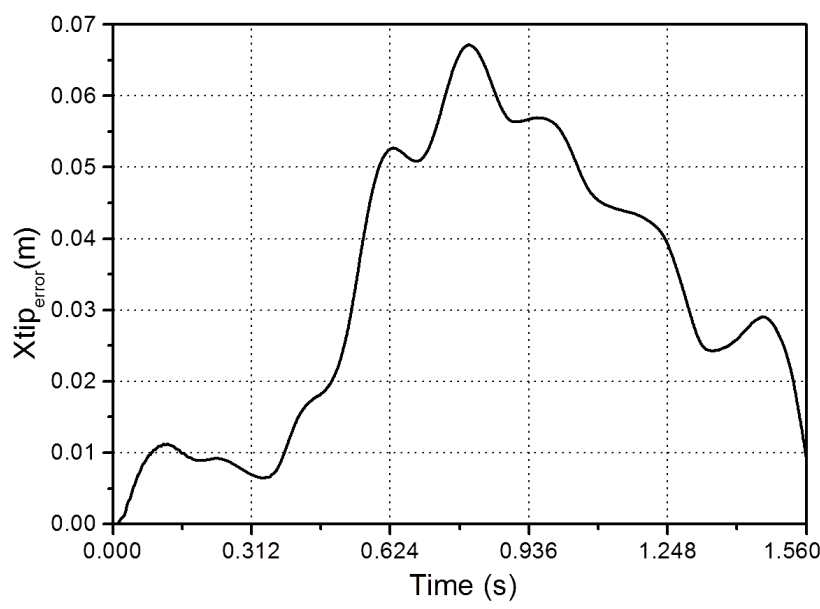

(a)

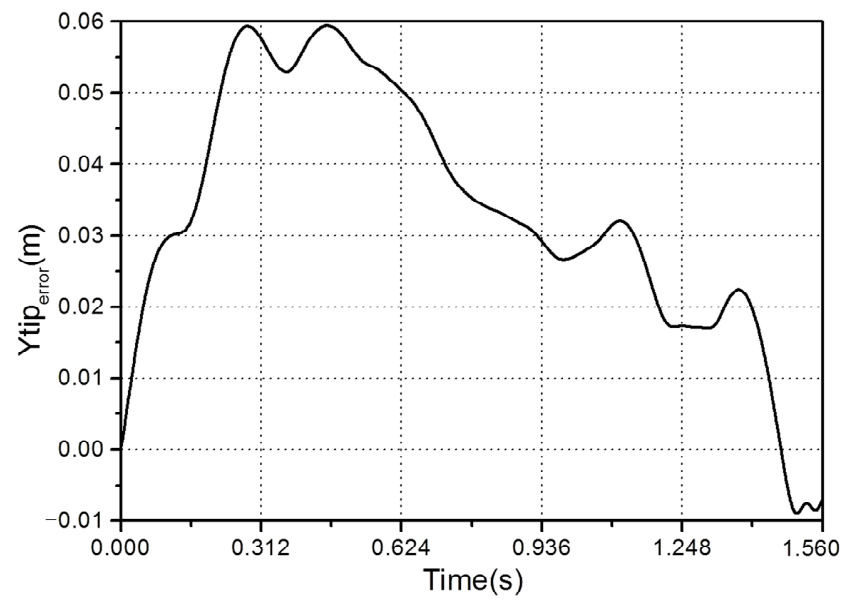

(b)

Figure 6. (a) Error in $X$ tip trajectory with respect to time; (b) Error in $Y$ tip trajectory with respect to time. 
of the reduced model based controller is that it does not require the link dynamic parameters and the trajectory tracking is very good. However the model has limitation as compared to analytical model as it is finite approximation of continuous system. The future work will be focus on developing a reduced model based controller for three dimensional flexible space manipulator.

\section{References}

[1] Y. Murotsu, S. Tsujio, K. Senda and M. Hayashi, "Trajectory Control of Flexible Manipulators on a Free Flying Space Robot," IEEE Control Systems, Vol. 12, No. 3, 1992, pp. 51-51. doi:10.1109/37.165517

[2] Y. Murotsu, K. Senda and M. Hayashi, "Some Trajectory Control Schemes for Flexible Manipulators on a Free Flying Space Robot," Proceedings of International Conference on Intelligent Robots and Systems, Raleigh, 1992.

[3] B. Samanta and S. Devasia, "Modeling and Control of Flexible Manipulators Using Distributed Actuators: A Bond Graph Approach," Proceedings of the IEEE International Workshop on Intelligent Robots and Systems, 1988. doi:10.1109/IROS.1988.592414

[4] B. Fardanesh and J. Rastegar, "A New Model Based Tracking Controller for Robot Manipulators Using the Trajectory Pattern Inverse Dynamics," Proceedings of the 1992 IEEE International Conference on Robotics and Automation, Nice, 1992. doi:10.1109/ROBOT.1992.219938

[5] F. Y. Wang and Y. Gao, "Advanced Studies of Flexible Robotic Manipulators: Modeling, Design, Control and Application: Series in Intelligent Control and Intelligent Automation," Word Scientific, New York, 2003.

[6] M. B. Leahy Jr., D. E. Bossert and P. V. Whalen, "Robust Model-Based Control: An Experimental Case Study," Proceedings of the 1990 IEEE International Conference on Robotics and Automation, Cincinnati, Vol. 3, 1990, pp. 1982-1987.

[7] K. Fawaz, R. Merzouki and B. Ould-Bouamama, "Model Based Real Time Monitoring for Collision Detection of an Industrial Robot," Mechatronics, Vol. 19, No. 5, 2009, pp. 695-704. doi:10.1016/j.mechatronics.2009.02.005

[8] S. K. Tso, L. Law, Y. Xu and H. Y. Shum, "Implementing Model-Based Variable-Structure Controllers for Robot Manipulators with Actuator Modeling," Proceedings of the 1993 IEEE/IRSJ International Conference on Intelligent Robots and Systems, Yokohama, 1993. doi:10.1109/IROS.1993.583137

[9] H. A. Zhu, C. L. Teo and G. S. Hong, "A Robust Model-Based Controller for Robot Manipulators," Proceedings of the IEEE International Symposium on Industrial Electronics, Vol. 1, 1992, pp. 380-384.

[10] Z. Qu, J. F. Dorsey, X. Zhang and D. M. Dawson, "Robust Control of Robots by the Computed Torque Law," Systems and Control Letters, Vol. 16, No.1, 1991, pp.25-32. doi:10.1016/0167-6911(91)90025-A

[11] P. K. Khosla and T. Kanade, "Real-Time Implementation and Evaluation of Computed-Torque Schemes," IEEE Transactions on Robotics and Automation, Vol. 5, No.2, 1989, pp. 245-263. doi:10.1109/70.88047

[12] P. M. Pathak, R. Prasanth Kumar, A. Mukherjee and A. Dasgupta, "A Scheme for Robust Trajectory Control of Space Robots," Simulation Modeling Practice and Theory, Vol. 16, 2008, pp. 1337-1349. doi:10.1016/j.simpat.2008.06.011

[13] R. M. Berger, H. A. EIMaraghy and W. H. EIMaraghy, "The Analysis of Simple Robots Using Bond Graphs," Journal of Manufacturing Systems, Vol. 9 No. 1, 2003, pp. 13-19, 2003.

[14] A. K. Samantaray and A. Mukherjee, "Users Manual of SYMBOLS Shakti," High-Tech Consultants, S.T.E.P., Indian Institute of Technology, Kharagpur, 2006. 